\title{
Investigating the impacts of street environment on pre-owned housing price in Shanghai using street-level images
}

\author{
Waishan Qiu', Xiaokai Huang ${ }^{2}$, Xiaojiang $\mathrm{Li}^{3}$, Wenjing $\mathrm{Li}^{4}$, Ziye Zhang \\ ${ }^{1}$ Department of City and Regional Planning, Cornell University, USA; ${ }^{2}$ Temple University, \\ USA; ${ }^{3}$ Graduate School of Design, Harvard University, USA; ${ }^{4}$ Center for Spatial Information \\ Science, The University of Tokyo, Japan; ${ }^{5}$ Cornell Institute for China Economic Research, \\ Cornell University, USA.
}

\begin{abstract}
Studies considering street environment quality's impact on housing value were limited to top-down variables such as the green ratio measured from satellite maps. In contrast, this study quantified street views' impacts on the value of second-hand commodity residential properties in Shanghai based on analysis of street view imagery. (1) It applied computer vision to objectively measure street features from largely accessible street view imagery. (2) Based on the classical urban design measures frameworks, it applied machine learning to evaluate human perceived street quality as street scores systematically, in contrast to the common practice of doing so in a more intuition-based fashion. (3) It further identified important indicators from both human-centered street scores as well as the more objective street feature measures with positive or adverse effects on property values based on a hedonic modeling method. The estimation suggested both street scores and features are significant and nonnegligible. For the perceived street scores (from 0-10 scale), neighborhoods with a unit increase in their "enclosure" or "safety" score enjoy price premium of $0.3 \%$ to $0.6 \%$. Meanwhile, streets with $10 \%$ greater tree canopy exposure are attributable to a $0.2 \%$ increase in the property value. This study enriched our current understanding at a micro level of the factors that impact property values from the perspective of the built environment. It introduced human-centered perception of street scores and objective measures of street features as spatial variables into the analysis of neighborhood attribute vectors.
\end{abstract}

Keywords: Machine learning; Property value; Shanghai; Street view imagery. 


\section{Introduction}

\subsection{Street Quality and Property Values}

The quality of streets is among those factors that have attracted attention in terms of its impact on the surrounding property values. Scholars have made many efforts to investigate the impact of streetscapes especially those urban trees on the property values. Most existing research focuses on objective metrics such as the numbers of trees, the size of tree covers, the distance to large green areas, or the total greenery measured from satellite images, failing to incorporate the importance of human perceptions. Killicoat et al. (2002) identified trees as important amenities creating an environment conducive to real estate transactions. Using a hedonic price model (HPM), Sander et al. (2010) implied that $10 \%$ more tree cover on parcels increased sales price by $0.48 \%$ in Minnesota. Besides trees, the impacts of other features on the property value, such as open space (Anderson \& West, 2006), street lighting (Willis et al., 2005), and surface street traffic (Larsen \& Blair, 2014) have been studied separately with the HPM approach.

\subsection{Street Environment Measures}

Physical features alone may not represent people's overall image of street quality, because the cumulative effect is greater than the total of each. Streetscape affecting perceived street environment quality should be defined and measured empirically (Yin, 2014). Cervero \& Kockelman (1997) first proposed the framework of 3Ds- density, diversity and design, and used gross quantities such as building height, block length and street width to measure the design quality of streets. However, people do not experience a street from these gross metrics. More operational definitions and measurement protocols about perceived quality were required (Brownson et al., 2004; Ewing \& Clemente, 2013a; Laaksonen et al., 2006; Pikora et al., 2003). Expert panels were recruited by Ewing et al. (2006) to rate five streetscape qualities based on video clips, namely imageability, visual enclosure, human scale, transparency and complexity. Physical features in the video clips were also quantified, and their relationships with the ratings from expert panels were statistically analyzed (Ewing et al., 2006). Conventional method such as visual collage or phone surveys to collect people's perceptions has many problems. First, the reliability of the operation is questionable (Nasar, 1990), as individual differences are one of the problems to make the evaluation impractical (Ewing \& Handy, 2009). Second, the low-throughput method results in constrained application to larger geographic contexts. Limited by the high survey cost or the poor spatial resolution, a segment of the city was often utilized as a sample area, making their conclusions subject to the particular conditions in that area and weak in generalizability (Seiferling et al., 2017). 


\subsection{Novel Streetscape Measures}

Most recently, studies in this regard started to exploit multisource open data and showed significant advantages in efficiency and accuracy over traditional methods. One example is the increasing use of the street view image (SVI). Differing from satellite imagery, SVI represents a ground-based perspective of urban streets and is available for the city wide (Seiferling et al., 2017). Compared to on-site auditing, the use of SVI was proved to be efficient for large-scale objective data collection (Griew et al., 2013; Rundle et al., 2011). Meanwhile, with the advance of artificial intelligence (AI), computer vision (CV) and machine learning (ML) algorithms were developed to predict human perception of the street environment from images. Naik et al. (2014) predicted the perceived safety score using labelled SVI as the training data from an online survey with more than 7000 participants. Chen et al. (2020) compared the non-linear relationship between housing prices and GVI in Shanghai and found higher GVI were significantly associated with more expensive houses.

Despite these meaningful explorations, our understanding of the impact of the street environment on economic outcomes is still limited by the drawbacks in the literature such as small samples, inconsistent ratings, and lack of human perception measures. This study aimed to investigate the impact of perceived streetscape on property values for the city-wide Shanghai, using a reliable and high-throughput approach with the advance of AI. It achieved at least four key contributions. First, it evaluated the feasibility of utilizing SVI and CV for auditing urban environments at a large scale, quantifying percentile index or counts for 30 street features with 25,276 SVIs. Second, it developed an online survey collecting the collaborative perceptions of street quality with 100 participants. Third, it trained efficient ML models to evaluate three important perceived street qualities, namely visual enclosure, complexity and aesthetics. Fourth, it identified the effects of perceived qualities and objective percentile index of streetscape with positive or adverse effects on property values using more than 40,000 housing transactions. By providing a more comprehensive understanding of the relationship between perceived streetscape qualities and property values in a city-wide scale, this study would inform policy-making and urban design.

\section{Data and Methods}

\subsection{Hedonic Price Model}

The hedonic price model (HPM) was widely adopted to investigate the impacts of built environment features on housing prices (Chen et al., 2020; Larsen \& Blair, 2014; Zhang \& Dong, 2018). It assumes that housing is a heterogeneous good, and the determinants of its price can be investigated by regressing the housing price on three types of independent variables capturing the property's structural, locational and neighborhood attributes (Rosen, 
1974). Our focus is on a subgroup of attributes relevant to the street quality, and thus we expand the conventional HPM as follows:

$$
\operatorname{lnPRICE}=\alpha+\beta 1 \text { STRU }+\beta 2 \text { LOCA }+\beta 3 \text { NEIG }+\beta 4 \text { STRE }+\varepsilon,(1)
$$

where $\ln$ PRICE was the natural logarithm of the transaction price; $\alpha$ is the regression constant; $\beta 1$ to $\beta 4$ are the estimated coefficients of the structural (STRU), locational (LOC), neighborhood (NEIG), and streetscape ((STRE)) attributes respectively; $\varepsilon$ is the error term.

\subsection{Data Collection and Processing}

\subsubsection{Housing Transactional Price}

This study collected 65,000 transaction records of preowned apartments occurring in 2019 within Shanghai from Lianjia.com. Besides the sales price, the data also included housing structure attributes and property's geolocation. The dataset was cleaned for (1) records whose price did not make sense, e.g. being zero or much higher or lower than the neighbors' mean; and (2) records whose property attributes were missing. 40,159 geo-tagged records were kept, with an average sales price at about $57,000 \mathrm{RMB} / \mathrm{m}^{2}$.

\subsubsection{Structural Attributes}

For structural attributes, selected continuous variables included floor area, numbers of bathroom, total numbers of floor, the year of construction. Categorical variables of relative floor level in the building, unit layout, building type, facing direction, structure type, interior decoration, availability of elevators were transformed to dummies.

\subsubsection{Locational Attributes}

Notably, a large body of literature have identified the pattern when distances to the city center increase, housing prices decrease (Bourassa et al., 2010; Chen et al., 2020; Rosen, 1974). We calculated the Euclidean distance from each property (1) to the Bund - CBD of Shanghai and (2) to their nearest county center as locational attributes.

\subsubsection{Neighborhood Attributes}

The neighborhood variables measured the (1) density, (2) distance to and (3) accessibility to urban services. The living amenities density was the number of services such as retailing, restaurants, cafes, groceries, hospitals and gyms per $\mathrm{km}^{2}$ within the neighborhood's administration boundary. Distances variables calculated the Euclidean distance to the closest metro station and school. Accessibility to metro stations and schools was also adopted and measured by the numbers of schools and metro stations can be reached to within 5 kilometer and 1 kilometers respectively. 


\subsubsection{Street environment features}

Motivated by the classical measurement protocols of Ewing et al. (2006), this study chose "visual enclosure" and "complexity", and modified the term "imageability" to "aesthetics" to present the perceived streetscape qualities in three different dimensions. Specifically, (1) visual enclosure refers to the extent streets are visually defined by features such as trees, walls and buildings; (2) complexity refers to the visual richness of a place, depending on the variety of and numbers of elements such as human activity, signage, street furniture, greenery, and buildings; (3) borrowing the term "imageability" which was a categorical variable, instead we used "aesthetics" to present the overall visual quality of a street being comfortable and distinct (Ewing et al., 2006). Meanwhile, many literatures identified urban trees as a significant factor affecting property value by using the percentile index GVI. We selected GVI as the objective measure, whose value was given by the ratio of pixels associated with tree canopy to the total pixels in an image:

GVI $=$ Pixels $s_{\text {greenery }} /$ Pixels $_{\text {total }}$,

The process of calculating these four variables from images involved five steps: (1) sampled SVI sites, (2) downloaded SVI, (3) collected people's collaborative ratings as training data's Y labels, (4) extracted pixel ratios of different streetscape features as training data's $\mathrm{X}$ variables, and (5) trained ML models and predicted perceived scores. First, given that many transactional data had the duplicated coordinates, its SVI data were sampled at 50-meter intervals along the centerline of all public streets within the property's 1-km radius. The sampling was processed in ArcGIS. In total, we sampled 30,000 SVI sites. Second, for each SVI sample site, its SVI was acquired by inputting its coordinates into Baidu Street View API. We controlled consistent camera settings, same size (600 by 300 pixels) and similar shooting time (summer and fall 2017) for each SVI to ensure the consistent perspective and to eliminate the seasonal variance in street environments (Fig.1b). Due to the limited data availability, not all sampled sites have SVI, as a result we downloaded 25,276 valid SVI. The values for the missing sample sites would be estimated through spatial interpolation detailed in the next section. 

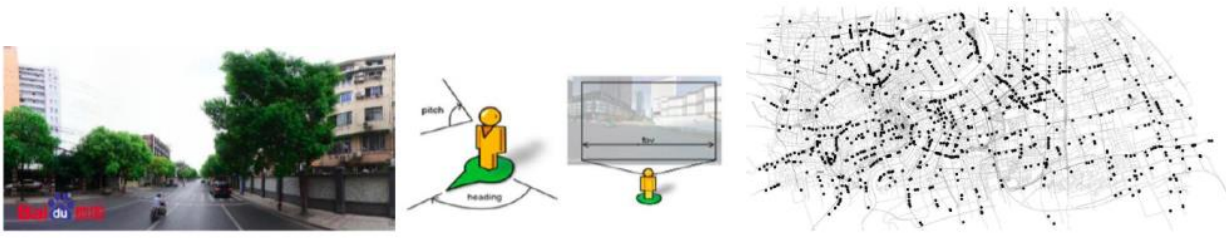

Figure 1. Downloading Baidu Street View Image.

(a) A typical SVI downloaded for this study, whose "point of view" was located in the middle of the road, being paralleled to the road's direction. (b) The camera settings were controlled by "heading", "FOV”, "pitch" and "resolution". In this study, the camera heading was set the same as the sample site's street center line tangent degree; the FOV (horizontal field of view) was set 120 degree, the pitch (specifies the up or down angle of the camera relative to the Street View vehicle) was 0 degree, and the resolution was $600 \times 300$ pixels, for all images. (c) Training images were sampled across a wide range of geographical locations in Shanghai.

Third, to acquire training data of people's preferences on streetscapes from SVI, we adopted the high-throughput method developed from emerging studies (Naik et al., 2014; Salesses et al., 2013). Specifically, we developed an online survey platform on which participants were shown two randomly chosen SVIs in Shanghai. Participants were asked to click on the SVI they preferred in response to three evaluative questions in regard to perceived street qualities, namely the Visual Enclosure, the Complexity and the Aesthetics. For example, for Visual Enclosure, we gave a brief definition on Visual Enclosure. Then participants were asked the question "Which place do you feel having a higher level of visual enclosure". Noted that, to ensure the training images would cover a wide range of streetscapes from city center, suburban to countryside, we randomly sampled 500 images across the whole region of Shanghai (Fig. 1c). In total 100 unique participants from Shanghai ranked the images using 8426 pairwise clicks. These preferences were converted to ranked scores for each image with Microsoft Trueskill algorithm (Naik et al., 2014). On average each unique image was compared to 16.8 other images, which was sufficient to lead Trueskill converge to stable ranked scores (Naik et al., 2014). The ranked scores were then normalized to a 0-10 scale. These 500 images with three ranked scores as labels, became the training data from which ML algorithms were trained to predict the collaborative perceptions of the streetscape. 

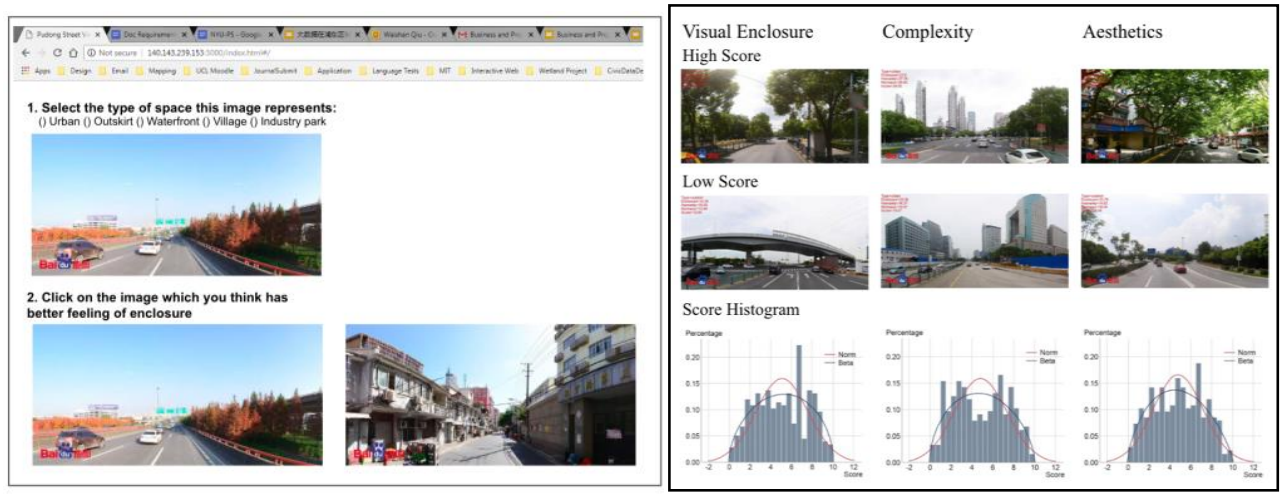

Figure 2. Collaborative Image of Streetscape with an Online Survey Platform (a) The survey system asked participants to choose from the pairwise SVIs in response to questions. (b) High score, low score example images, and the histogram of score distribution, for each of the three perceived street qualities. From the high and low score examples, people seemed to prefer streetscapes with less sky exposure and more greenery.
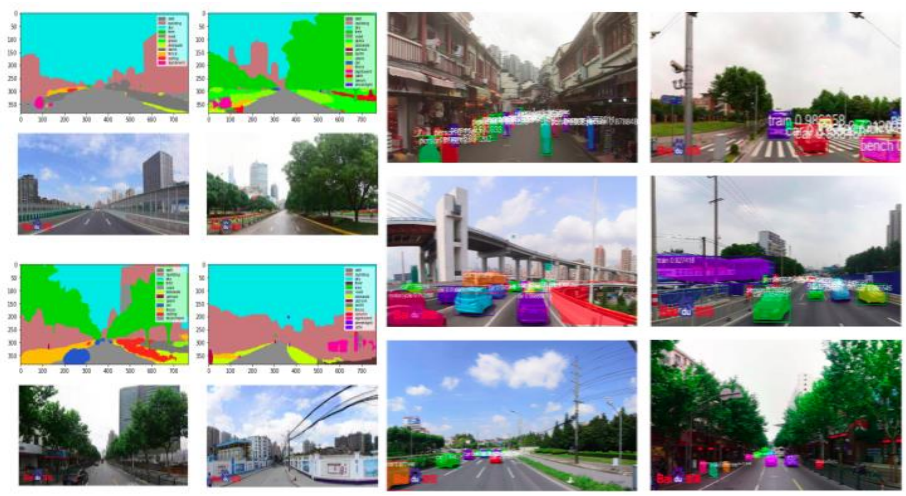

Figure 3. CV Segmentation Results (a) Pairwise PSPNet semantic segmentation results and its input SVI (b) Mask $R$-CNN instance segmentation results counting numbers of streetscape objects appearing in the SVI

Fourth, we quantified the pixel ratios or amounts of 30 street features from the 500 training images using pre-trained CV models. These features, including the building, sky, tree, curbs, roads, grass, glass and others, were conceived significant effects on human perceived street qualities by Ewing et al. (2006). On one hand, we applied Pyramid Scene Parsing Network (PSPNet) to calculate the pixel ratios of 30 kinds of features (Zhao et al., 2016). On the other hand, for five of the features, namely the car, people, bike, motorbike and chairs, an indicator of pixel ratio makes less sense than the absolute counts. So we applied pre-trained Mask RCNN to count their amounts (He et al., 2017). These 30 features became the explanatory variables to predict three perceived scores in the next step. 
Fifth, we trained ML models including K-nearest neighbors (KNN), support vector machine (SVM), random forest (RF), decision tree, and gradient boost to predict the three perceived scores from the 30 features extracted from SVI. The 500 labelled images from the online survey were split to 400 and 100 for training and testing purposes. To choose the optimal model, we compared model performances in regard to the R-square, the root mean square error (RMSE), and the mean absolute error (MAE). SVM outperformed other models in predicting enclosure and complexity score, while RF performed best in predicting aesthetics score. Regarding the accuracy, SVM predicted enclosure and complexity scores with an MAE of 1.51 and 1.35 respectively, while RF predicted aesthetics score with a 1.19 MAE (Table 3). The accuracy of ML model was considered acceptable, limiting by the small training dataset, the error magnitude of 1.2 to 1.51 would not offset predicted scores far from true value with regard to the $0-10$ scale scoring system. The global importance of individual streetscape features were compared using Python Scikit-learn's Tree Based Regressor. The algorithm computes how much each variable contributes to decreasing the weighted impurity during the training, thus providing the importance score (Chen et al., 2020; Naik et al., 2014). Pixels of trees ranked second, justified that GVI was an appropriate variable to represent the objective measure of streetscape.

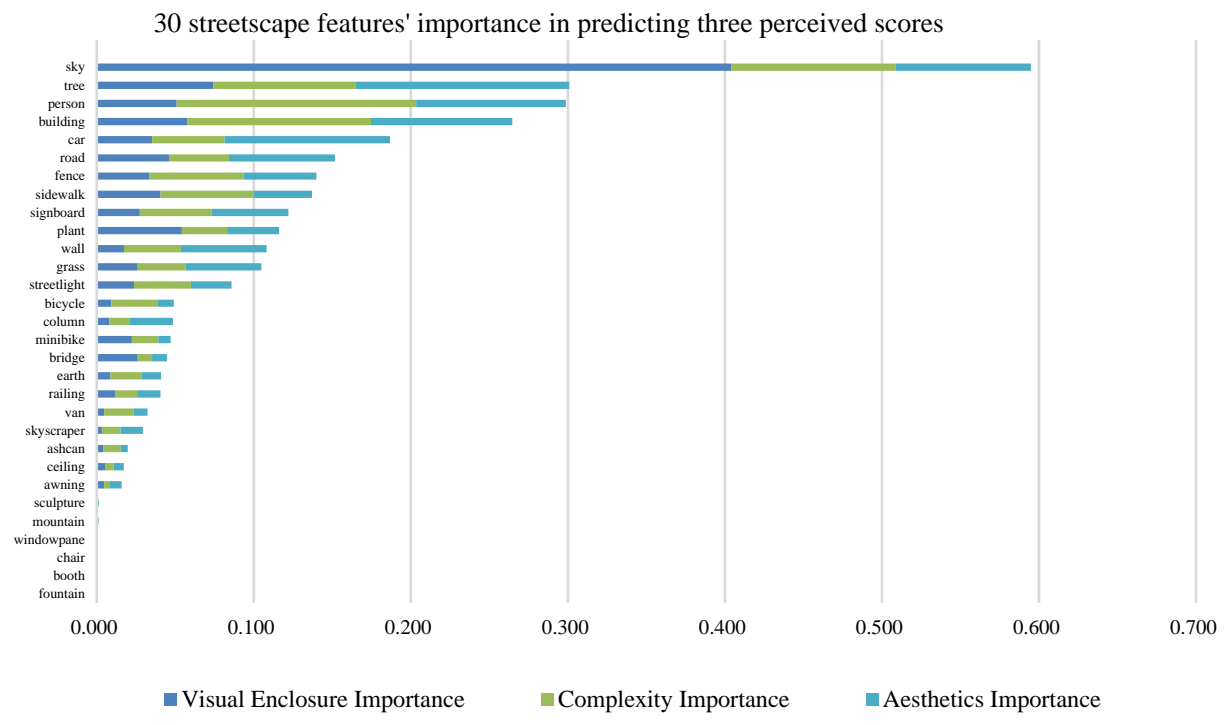

Figure 4. Feature importance in predicting perceived scores. 


\section{Reference}

Anderson, S. T., \& West, S. E. (2006). Open space, residential property values, and spatial context. Regional Science and Urban Economics, 36(6), 773-789. https://doi.org/10.1016/j.regsciurbeco.2006.03.007

Bourassa, S. C., Cantoni, E., \& Hoesli, M. (2010). Predicting House Prices with Spatial Dependence: A Comparison of Alternative Methods. Journal of Real Estate Research, 32(2), 139-160.

Brownson, R. C., Chang, J. J., Eyler, A. A., Ainsworth, B. E., Kirtland, K. A., Saelens, B. E., \& Sallis, J. F. (2004). Measuring the Environment for Friendliness Toward Physical Activity: A Comparison of the Reliability of 3 Questionnaires. American Journal of Public Health, 94(3), 473-483. https://doi.org/10.2105/AJPH.94.3.473

Brownson, R. C., Hoehner, C. M., Day, K., Forsyth, A., \& Sallis, J. F. (2009). Measuring the built environment for physical activity: state of the science. American Journal of Preventive Medicine, 36(4 Suppl), S99-S123.e12. https://doi.org/10.1016/j.amepre.2009.01.005

Cervero, R., \& Kockelman, K. (1997). Travel demand and the 3Ds: Density, diversity, and design. Transportation Research Part D: Transport and Environment, 2(3), 199-219. https://doi.org/10.1016/S1361-9209(97)00009-6

Chen, L., Yao, X., Liu, Y., Zhu, Y., Chen, W., Zhao, X., \& Chi, T. (2020). Measuring Impacts of Urban Environmental Elements on Housing Prices Based on Multisource Data-A Case Study of Shanghai, China. ISPRS International Journal of Geo-Information, 9(2), 106. https://doi.org/10.3390/ijgi9020106

Donovan, G. H., \& Butry, D. T. (2010). Trees in the city: Valuing street trees in Portland, Oregon. Landscape and Urban Planning, 94(2), 77-83. https://doi.org/10.1016/j.landurbplan.2009.07.019

Dubé, J., \& Legros, D. (2014). Spatial econometrics and the hedonic pricing model: what about the temporal dimension? Journal of Property Research, 31(4), 333-359. https://doi.org/10.1080/09599916.2014.913655

Ewing, R., \& Clemente, O. (2013). Measuring Urban Design: Metrics for Livable Places. Island Press/Center for Resource Economics. https://www.springer.com/gp/book/9781610912099

Ewing, R., \& Handy, S. (2009). Measuring the unmeasurable: Urban design qualities related to walkability. Journal of Urban Design, 14(1), 65-84.

Ewing, R., Handy, S., Brownson, R. C., Clemente, O., \& Winston, E. (2006). Identifying and Measuring Urban Design Qualities Related to Walkability. Journal of Physical Activity \& Health, 3(s1), S223-S240. https://doi.org/10.1123/jpah.3.s1.s223

Griew, P., Hillsdon, M., Foster, C., Coombes, E., Jones, A., \& Wilkinson, P. (2013). Developing and testing a street audit tool using Google Street View to measure environmental supportiveness for physical activity. International Journal of Behavioral Nutrition and Physical Activity, 10(1), 103. https://doi.org/10.1186/1479-5868-10-103

Hara, K., Sun, J., Moore, R., Jacobs, D., \& Froehlich, J. (2014). Tohme: detecting curb ramps in google street view using crowdsourcing, computer vision, and machine learning. 
Proceedings of the 27th Annual ACM Symposium on User Interface Software and Technology - UIST '14, 189-204. https://doi.org/10.1145/2642918.2647403

Kelly, C. M., Wilson, J. S., Baker, E. A., Miller, D. K., \& Schootman, M. (2012). Using Google Street View to audit the built environment: inter-rater reliability results. Annals of Behavioral Medicine, 45(suppl_1), S108-S112.

Killicoat, P., Puzio, E., \& Stringer, R. (2002). The economic value of trees in urban areas: estimating the benefits of Adelaide's street trees. Proceedings Treenet Symposium, 94, 106.

Laaksonen, P., Laaksonen, M., Borisov, P., \& Halkoaho, J. (2006). Measuring image of a city: A qualitative approach with case example. Place Branding, 2(3), 210-219. https://doi.org/10.1057/palgrave.pb.5990058

Larsen, J. E., \& Blair, J. P. (2014). Price effects of surface street traffic on residential property. International Journal of Housing Markets and Analysis. https://doi.org/10.1108/IJHMA-12-2012-0062

Lin, L., \& Moudon, A. V. (2010). Objective versus subjective measures of the built environment, which are most effective in capturing associations with walking? Health \& Place, 16(2), 339-348. https://doi.org/10.1016/j.healthplace.2009.11.002

Li, X., Zhang, C., Li, W., Ricard, R., Meng, Q., \& Zhang, W. (2015). Assessing street-level urban greenery using Google Street View and a modified green view index. Urban Forestry \& Urban Greening, 14(3), 675-685. https://doi.org/10.1016/j.ufug.2015.06.006

Naik, N., Philipoom, J., Raskar, R., \& Hidalgo, C. (2014). Streetscore -- Predicting the Perceived Safety of One Million Streetscapes. 2014 IEEE Conference on Computer Vision and Pattern Recognition Workshops, 793-799. https://doi.org/10.1109/CVPRW.2014.121

Nasar, J. L. (1990). The Evaluative Image of the City. Journal of the American Planning Association, 56(1), 41-53. https://doi.org/10.1080/01944369008975742

Orland, B., Vining, J., \& Ebreo, A. (1992). The Effect of Street Trees on Perceived Values of Residential Property. Environment and Behavior, 24(3), 298-325. https://doi.org/10.1177/0013916592243002

Pandit, R., Polyakov, M., \& Sadler, R. (2014). Valuing public and private urban tree canopy cover. Australian Journal of Agricultural and Resource Economics, 58(3), 453-470. https://doi.org/10.1111/1467-8489.12037

Pikora, T., Giles-Corti, B., Bull, F., Jamrozik, K., \& Donovan, R. (2003). Developing a framework for assessment of the environmental determinants of walking and cycling. Social Science \& Medicine, 56(8), 1693-1703. https://doi.org/10.1016/S02779536(02)00163-6

Rosen, S. (1974). Hedonic Prices and Implicit Markets: Product Differentiation in Pure Competition. Journal of Political Economy, 82(1), 34-55. JSTOR.

Rundle, A. G., Bader, M. D. M., Richards, C. A., Neckerman, K. M., \& Teitler, J. O. (2011). Using Google Street View to Audit Neighborhood Environments. American Journal of Preventive Medicine, 40(1), 94-100. https://doi.org/10.1016/j.amepre.2010.09.034 
Salesses, P., Schechtner, K., \& Hidalgo, C. A. (2013). The Collaborative Image of The City: Mapping the Inequality of Urban Perception. PLOS ONE, 8(7), e68400. https://doi.org/10.1371/journal.pone.0068400

Sander, H., Polasky, S., \& Haight, R. G. (2010). The value of urban tree cover: A hedonic property price model in Ramsey and Dakota Counties, Minnesota, USA. Ecological Economics, 69(8), 1646-1656. https://doi.org/10.1016/j.ecolecon.2010.03.011

Seiferling, I., Naik, N., Ratti, C., \& Proulx, R. (2017). Green streets- Quantifying and mapping urban trees with street-level imagery and computer vision. Landscape and Urban Planning, 165, 93-101.

Willis, K. G., Powe, N. A., \& Garrod, G. D. (2005). Estimating the Value of Improved Street Lighting: A Factor Analytical Discrete Choice Approach. Urban Studies, 42(12), 22892303. https://doi.org/10.1080/00420980500332106

Yin, L. (2014). Book Review: Measuring Urban Design: Metrics for Livable Places. Journal of Planning Literature, 29(3), 273-274. https://doi.org/10.1177/0885412214531541

Yin, L., \& Wang, Z. (2016). Measuring visual enclosure for street walkability: Using machine learning algorithms and Google Street View imagery. Applied Geography, 76, 147-153. https://doi.org/10.1016/j.apgeog.2016.09.024

Zhang, Y., \& Dong, R. (2018). Impacts of Street-Visible Greenery on Housing Prices: Evidence from a Hedonic Price Model and a Massive Street View Image Dataset in Beijing. ISPRS International Journal of Geo-Information, 7(3), 104. https://doi.org/10.3390/ijgi7030104 